\title{
Review of Family Functioning
}

\author{
Liangtie Dai, Lingna Wang \\ School of Management, Jinan University, Guangzhou, China \\ Email: WANGLINGNA927@163.com
}

Received 25 November 2015; accepted 19 December 2015; published 22 December 2015

Copyright (C) 2015 by authors and Scientific Research Publishing Inc.

This work is licensed under the Creative Commons Attribution International License (CC BY).

http://creativecommons.org/licenses/by/4.0/

(c) (i) Open Access

\begin{abstract}
This article introduces the theories of family functions, including two kind of theory. The first is result oriented, defining family functioning by specific features of family. The second is process oriented, describing family function from the tasks families need to complete. The authors review Olson annular mode theory and Beavers system theory as representatives of result oriented family function. Then, the authors introduce McMaster family functioning mode theory and Skinner's Family process model theory as representatives of process oriented family function. At the end of this paper, the authors analyze some possible research directions in the future.
\end{abstract}

\section{Keywords}

Family Functioning, Result Oriented Family Function, Process Oriented Family Function, Measure

\section{Introduction and Overview}

Family is not only the basic unit of society, but also an important place for individual physical and mental growth. A family is made up of every member in the family. At the same time, it affects the growth of every member, and plays an important role in the normal operation process of social system. Therefore, family function is restricted by the characteristics of the family itself, and depends on the social demand. The concept of family functioning, which embodies the characteristics of the family as a system, has been proposed by scholars since the 1970s. So, the concept gets the favour of many researchers. More and more researchers gradually turned to the study of family functioning instead of exploring one or some family factors research paradigm.

At present, the domestic and foreign researchers of family functioning form two main theories: The first is result oriented, defining family functioning by specific features of family. The second is process oriented, describing family function from the tasks families need to complete.

In this article, the authors summarize the theories of family functioning and form a new combination, which is innovative. For the purpose of reviewing the theories and indicating new directions, the authors first introduce two kinds of theories of family functions, then propose a new family function theory by combining the two 
kinds of family functions, describe some questionnaires which measure family function, summarize factors which influence family function, and propose directions for future research in this field.

\section{Result Oriented Family Function}

The representatives of this theory are Olson annular mode theory and Beavers system theory. Family was divided into different types mainly according to the results of family function played, to distinguish which belongs to the healthy development and which needs family therapy and intervention.

\subsection{Olson Annular Mode Theory}

This theory was put forward in 1978, based on the theory of family system, mainly used in family studies, clinical evaluation, training, marriage and family therapy. Olson annular mode theory is the most widely used in the field of family function theory.

Olson's family function theory can be divided into three dimensions: family intimacy, family adaptability and family communication. The family intimate degree refers to the relationship between family members; Family adaptability refers to the family need to change the power structure, role assignment, or the ability of family rules to cope with the external environmental pressure or the development of marriage; Family communication refers to the communication between family members, which is important for the development of family intimacy and adaptability.

Olson annular mode theory divides family intimacy into four levels from low to high: disengaged, separated, connected and enmeshed. Olson annular mode theory divides family adaptability four levels from low to high: rigid, structured, flexible and chaotic. Two dimensions of four level forms 16 kinds of family. The theory Originally hypothesis that there is a curve linear relationship between the result of family function and its two dimensions. Too high or too low scores hindered the function of the family. So Olson divides the 16 kinds of family into three types: balance type, middle type and extreme type.

The balanced type of 4 kinds of family is characterized by moderate on two dimensions, which adapts well. Moderate score on one dimension, but extreme score for another dimension of 8 kinds of family are called the middle type. The extreme type is characterized by extreme on two dimensions of 4 kinds of family, such family and its members are often maladjustment.

The authors of this article summarize the theory into Table 1.

Olson adjusts five levels for each dimension in 2003. Olson increased a middle level respectively in the two dimensions of the original level, into five levels, so that the amount of family type increases into 25 [1]. In the new theory, the balanced type increases into 9 kinds of family; the middle type increases into 12 kinds of family; the extreme type remains to be 4 kinds of family.

\subsection{Beavers System Theory}

Beavers system model theory is proposed mainly by Beavers and others in 1977 [2], to examine the relationship between the family functions and the strain capacity of family system.

\subsubsection{The Specific Content of the Theory}

Beavers system model theory examine family functioning from two dimensions: one is the rigid index, including family structure, family relationship and family reaction force. There is a linear relationship between these characteristics and the effect of family function. That is to say, the more flexible the rigid index is, the better the

Table 1. The structure of Olson annular mode theory.

\begin{tabular}{clll}
\hline \multicolumn{1}{c}{ Intimacy } & Disengaged & Separated & Connected \\
\hline Adaptability & Extreme type & Middle type & Middle type \\
Rigid & Middle type & Balanced type & Balanced type \\
Flexible & Middle type & Balanced type & Balanced type \\
Chaotic & Extreme type & Middle type & Middle type \\
\hline
\end{tabular}


family functioning plays. The other is the soft index, referring to family member's communication style. There is a nonlinear relationship between the effect of family function and family member's communication style, namely inverted u-shaped relationship. Only when communication style is in the intermediate state, it is conducive to family function, at the two extreme states of centripetal and centrifugal model family will appear to adjustment disorder of communication.

Behavers divides family into five types according to rigid index of family function: serious obstacle, borderline, intermediate, appropriate and best. Among them, the appropriate and best family belongs to healthy family types, and the rest three types of family encounter family functional problems.

Then on the basis of family soft data (family member's communication style), intermediate type is subdivided into three kinds: the centripetal intermediate family, the centrifugal intermediate family and hybrid intermediate family. On the basis of family soft data, borderline type is subdivided into two kinds: the centripetal borderline family and the centrifugal borderline family. On the basis of family soft data, serious obstacle type is subdivided into two kinds: the centripetal serious obstacle family and the centrifugal serious obstacle family.

In all, Beavers system model divides family into 9 types according the relationship between the family functions and the strain capacity of family system: the centripetal serious obstacle family, the centrifugal serious obstacle family, the centripetal borderline family, the centrifugal borderline family, the centripetal intermediate family, the centrifugal intermediate family, the hybrid intermediate family, the appropriate family and the best family.

The authors of this article summarize the theory into Table 2.

\subsubsection{The Application of Theory}

Then, the authors summarize the application of Beavers system theory from the view of every type of family. The character for every type of family is as following.

Appropriate and best family rarely encounter extreme communication mode, so that they belong to healthy family.

Intermediate type includes three kinds of family: the centripetal intermediate family, the centrifugal intermediate family and hybrid intermediate family. The centripetal intermediate family tends to have relatively fixed family relation mode, such as autocratic father or blindly obey mother. In order to maintain the inherent relationship in the family, family members often have to bear huge psychological pressure, resulting in forming the parenting patterns with neurotic traits. The centrifugal intermediate family lack relatively stable patterns of human relationships, so this kind of family often break out agonistic behaviors. Family members in this kind of family are weak in controlling others and themselves. The hybrid intermediate family is more flexible in terms of communication, and the ability to adapt is relatively good.

Borderline type includes two kinds of family: the centripetal borderline family and the centrifugal borderline family. The centripetal borderline family takes strict and depressive restrictions against members' agonistic behaviors, which is easy for family member to form compulsive behavior or compulsive character. The centrifugal borderline family often quarrels, and in their children's education requirement the family is not consistent. The child tends to be unstable on emotion and personality traits.

Serious obstacle type includes two kinds of family: the centripetal serious obstacle family and the centrifugal

Table 2. The structure of Beavers system theory.

\begin{tabular}{|c|c|c|c|c|}
\hline $\begin{array}{l}\text { Rigid index } \\
\text { Soft index }\end{array}$ & $\begin{array}{l}\text { Serious obstacle } \\
\text { family }\end{array}$ & Borderline family & Intermediate family & $\begin{array}{l}\text { Appropriate } \\
\text { family }\end{array}$ \\
\hline Centripetal family & $\begin{array}{l}\text { The centripetal } \\
\text { serious obstacle } \\
\text { family }\end{array}$ & $\begin{array}{l}\text { The centripetal } \\
\text { borderline family }\end{array}$ & $\begin{array}{c}\text { The centripetal } \\
\text { intermediate family }\end{array}$ & \multirow{3}{*}{ Healthy family } \\
\hline Hybrid family & & & $\begin{array}{l}\text { The hybrid } \\
\text { intermediate family }\end{array}$ & \\
\hline Centrifugal family & $\begin{array}{l}\text { The centrifugal } \\
\text { serious obstacle } \\
\text { family }\end{array}$ & $\begin{array}{l}\text { The centrifugal } \\
\text { borderline family }\end{array}$ & $\begin{array}{c}\text { The centrifugal } \\
\text { intermediate family }\end{array}$ & \\
\hline
\end{tabular}


serious obstacle family. The centripetal serious obstacle family claim members' behavior and role to be too stiff, requires each member to be loyal unconditionally. This kind of family emphasizes the intimacy among family members and often ignores the need of individuals on psychology and emotion. The centrifugal serious obstacle family looks like a fierce "battlefield", which often arises fierce verbal or physical conflict. As a result, there are often family members away from home. This kind of family shows rejection and cavalier attitude to depending on each other and the tender care.

The difference between the above 9 types of family is relative. The family's type doesn't always remain the same. One family may show the typical characteristics of different types in different stages of development.

\section{Process Oriented Family Function}

The representatives of this theory are McMaster family functioning mode theory and Skinner's process oriented family function. They think family system affect physical and psychological health of individual not by the family structure characteristics, but by the process realizing various functions in the family. The more smoothly family to realize its function process, the healthier the family members are in mentality.

\subsection{McMaster Family Functioning Mode Theory}

McMaster family functioning model theory is proposed by Epstein in 1987 [3]. He regards the process of the family system as the core, and assumes that the basic function of family is to provide appropriate environmental conditions for family members to develop on the physical, psychological, social and other aspects. To realize the basic function, the family system must complete a series of tasks, including basic tasks (such as meeting the material needs of the individual in terms of food and clothing living line), developmental tasks (such as adapting and promoting the growth and development of the members) and crisis tasks (such as dealing with all kinds of family emergencies).

The family system promotes the development of family and its members together by completing a series of family tasks. This theory divides family into six kinds by the ability of family realizing the basic function and the ability of completing the basic tasks. The six kinds respectively are: 1) Problem solving. In order to maintain effective family function, family solves problems which threaten family completely and functional capacity problem. 2) Communication. The information communication between family members can be known directly at any time. 3) Family role refers to the behavior patterns established by the family members to complete family function. 4) Affective response refers to the degree of emotional response of the family members to stimuli. 5) Affective involvement refers to the degree of concern and attention of family members to the activities and some other things among each other. 6) Behavior control refers to different behavior control mode in different situation of a family. Based on the above six ability of performance, McMaster family functioning mode theory investigates whether family functions well.

The authors of this article summarize the theory into Figure 1.

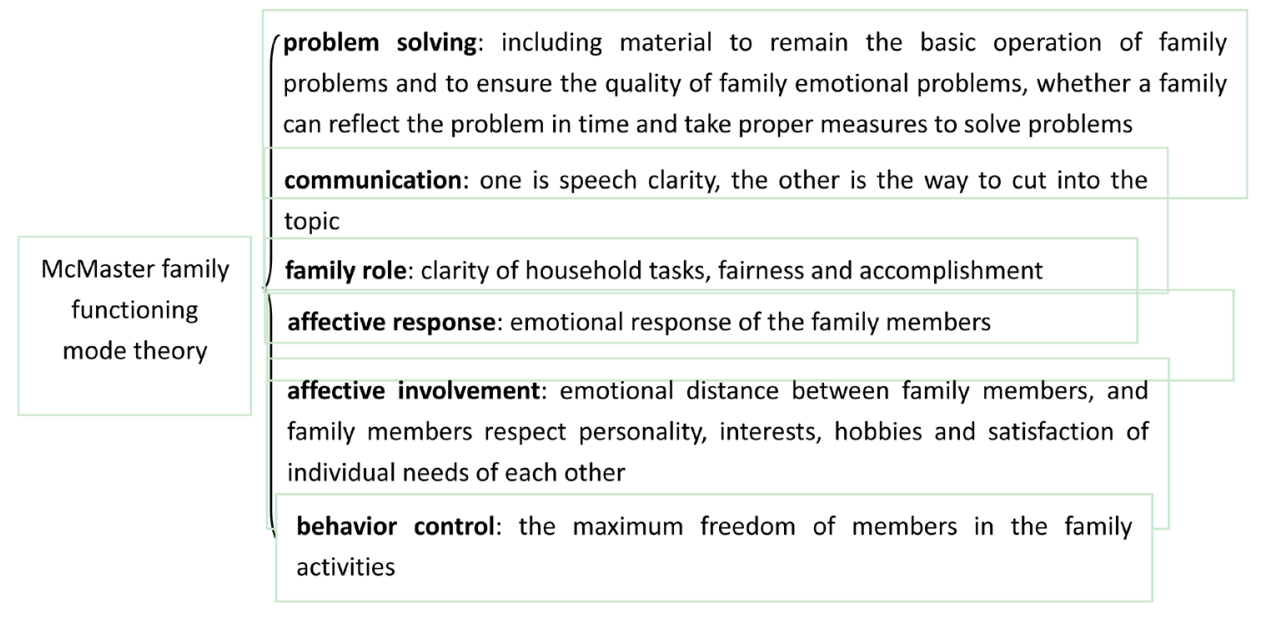

Figure 1. The structure of McMaster family functioning mode theory. 


\subsection{Skinner's Family Process Model Theory}

Family process model theory is proposed by Skinner in 1980 [4], which emphasize the interaction relationship between individual (family) and the overall (family). This theory uses concepts of McMaster family functioning mode theory to form a more systematic and clear function structure of family. At the same time, Skinner regard family as a dynamic operation system, and consider family background factors such as member of the family values and rules.

Family process model theory believes that, family function is embodied by family members play all kinds of daily tasks and deal with all kinds of family problems orderly and flexibly .In the process of each function being played, family and its members get growth too.

On this basis, the theory examines family function from seven dimensions: completion of task, role, communication, emotional expression, involvement, behavior and values and rules. The completion of task is the core dimension, including problem identification, considering all kinds of solution to the problem, selecting the appropriate solution and implementing it and evaluating the effectiveness of the solution. The rest six dimensions around the core dimension lead the team to complete this task.

First of all, in order to complete all the family tasks, family members need to undertake the various roles and responsibility in the task. In the process of role assignment ,family needs to communicate and coordinate .Then, in communication, the emergence of emotional expression will promote or hinder the role taking, during which mood will maintain all the period. Each family member's involvement and focus on tasks affect the completion. Behavior control is the behavior interaction of family members in the task completing process. Finally, in order to finish the task, family background factors influence the ways and rules of family members to complete the task. The interaction among seven dimensions make a family fully play its function of family and adapt changes between the various tasks.

The dimension of family values and rules is the new dimension added, refers to values and rules of completing family task.

The authors of this article summarize the theory into Figure 2.

\section{Update of Family Function Theory}

The authors of this article summarize the theories above, and think that there is common ground among the theories. So the authors of this article combine the Result oriented family function and Process oriented family function into a new one. Perhaps, the new theory is not perfect and much room can be improved. So, the authors are looking forward to criticisms from other researchers.

Update family function theory consists of two dimensions: soft index and rigid index. Then the authors divide the process of family function into the two dimensions. Soft index includes affective responses, involvement, behavior control and values and rules. Regid index includes problem solving, communication and role assignment.

The authors of this article summarize the theory into Figure 3.

Skinner's Family
process model
theory $\left\{\begin{array}{l}\text { Task comleting } \\ \text { role } \\ \text { communication } \\ \text { emotional expression } \\ \text { involvement } \\ \text { behavior } \\ \text { values and rules }\end{array}\right.$

Figure 2. The structure of Skinner's family process model theory. 


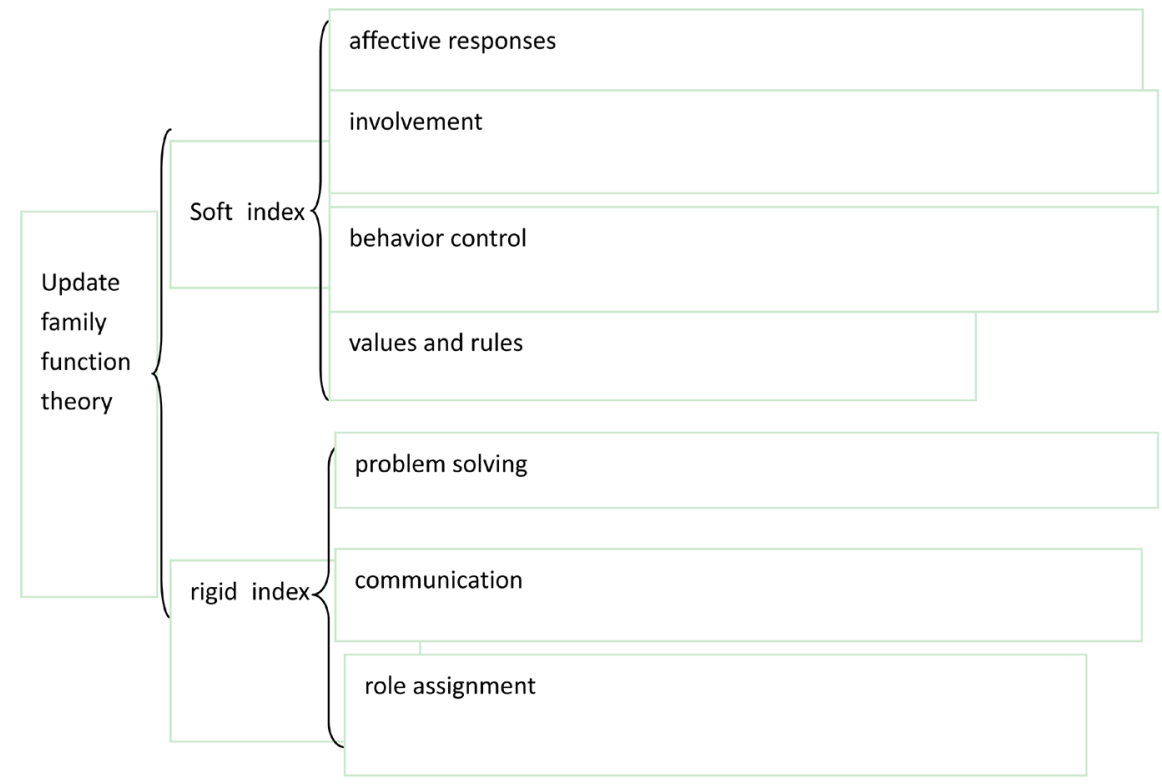

Figure 3. Update family function theory.

\section{Measure of Family Function}

The measure of family function focuses on questionnaire survey. The authors summarize 10 common questionnaires of this field.

\subsection{Family Assessment Device (FAD) Survey}

This scale includes 60 items [5], containing seven dimensions: problem solving, communication, role function, affective responses, involvement, behavior control and overall function. The scale corresponds to McMaster family functioning mode theory.

\subsection{Family Environment Scale (FES)}

The scale is divided into 10 dimensions, respectively evaluating 10 different family social and environmental characteristics. The scale consists of a total number of 90 true-false questions, with a time limit of approximately 30 minutes.

10 dimensions respectively are cohesion, expressiveness, conflict, independence, achievement Orientation, intellectual-Cultural Orientation, active-Recreational Orientation, Moral-Religious Emphasis, organization, and control.

\subsection{The Family Concern Index Questionnaire (APGAR)}

The scale is widely used in the public medical institutions research on the relationship between the family functioning and health problems [6], and used to assess satisfaction of family adults with social support. The scale consists of 5 items for received support of family members in 5 different fields. The 5 fields are adaptation, cooperation, growth, emotion, and problem solving.

\subsection{The Family Impact Scale (FIS)}

The scale consists of 14 items, trying to understand the influence of children's oral and written terms to 4 aspects: Parents and family activities, Parents' emotion, family conflict and family assets.

\subsection{The Family Assessment Measure (FAM)}

The FAM assessment includes 7 basic concepts: completion of task, role, communication, emotional expression, 
involvement, behavior and values and rules.FAM measure these dimensions on three levels: 1) regard family as a system of general scale (50 items), 2) examine the binary relation between couple scale (42 items), 3) test the individual function in the family (42 items). Each scale provides a different perspective of family functioning.

\subsection{The Family Functioning Index (FFI)}

The scale consists of 15 items, designed to measure family interaction of these areas: Marital satisfaction, frequency of divergence, communication, problem solving, and happiness and intimacy.

\subsection{The Family Functioning Questionnaire (FFQ)}

The scale measures psychosocial health of the families with young children. The scale examines six dimensions of family functioning: structure, emotion, communication, behavior control, Value Transmission and outer system.

\subsection{The Family Impact Questionnaire, Revised (FIQ-R)}

The scale assesses the influence of parents to children through several dimensions of family functions. It is adapted to 3.5 to 6 years old children. To be specific, they are parental and family activities (5 questions), parental emotions (4 questions), family conflict (4 questions) and family finances (1 question).

\subsection{The Family Life Questionnaire}

The scale consists of 14 items, including 4 subscales, namely, they are affirmation, punishment, special allowance and rule.

\subsection{The Chinese Version of the Feetham Family Functioning Survey (Chinese FFFS)}

The scale consists of 25 items, including 3 dimensions. It is used to measure family function, namely the relationship between Family and social unit (community, school, etc.), the relationship between family and the subsystem in the family, and the relationship between family and individual.

\section{The Influence Factors of Family Functioning}

Many factors can influence the family function, in this chapter, the authors summarize these factors.

First of all, the family structure influences the family function. Some family structure is single-parent, others are complete family [7].

The second factor is the social and economic status of the family. Families are different in many aspects, which influence family function, such as Monthly income, economic condition, Father's Occupation, Father's cultural degree and living condition.

What's more, the relationship of family members is also very important to the family function. The relationship involves parent-child relationship, spousal relationship and other interaction among family members, such as parenting way of family and so on.

Another factor is the stage of family. According to time period a family can belongs to different stages, such as young married with no child, phase with pre-school children, phase with elementary school children, phase with teenagers, the midstream of life, empty nest stage and the retirement years.

The last but not the least, Life events can also influence the family function [8]. There are many events that can influence our lives. For example, getting married, being fired, starting the employment, getting first child and so on.

\section{Prospect of the Family Functioning}

Depending on the summary above, the authors find that there are many theories in this field. In the future, there will be many new directions for scholars to research. The directions are found by the authors who need much room to progress. The authors are looking forward to suggestions and criticism.

First of all, the scholars in this field examine the theory just in one style. As the authors introduced in chapter 4, the two kinds of theories can be combined into the integrated one. Each theory has its advantage. At the same 
time, every theory can't cover all the aspects of family function. If the researchers try to combine all the different theories into a new one, perhaps it is beneficial for the future.

Secondly, family is closely connected with culture. So the cultural difference is very important for the future research. In china, the family functioning theory is focused on translated literature, but maybe these are not fit for Chinese. So in the future, Chinese researchers can develop some unique theories for the native.

Thirdly, the theories just introduced the idle state of family function, but not the development of the family function of different periods. So a new direction is time study. The scholars can study different types of family functions during the life of a family.

Finally, the theory just analyzed different types of family functions, but all theories didn't propose any treatment behavior for the family. In the future, researchers can offer some advice for the unhealthy families to develop smoothly.

\section{Acknowledgements}

First of all, I would like to extend my sincere gratitude to my supervisor, Liangtie Dai, for his instructive advice and useful suggestions on my thesis. I am deeply grateful of his help in the completion of this thesis. Second, I thank all the other tutors and teachers in my major for their effort. Finally, I am indebted to my parents and friends for their continuous support and encouragement.

\section{Project}

DBA120178, Key Project of Ministry of Education, “An empirical comparison of three typical psychotherapies in college students' internet addiction".

\section{References}

[1] Walsh, F. (2003) Normal Family Processes. 3rd Edition, Guilford, New York, 514-547. http://dx.doi.org/10.4324/9780203428436

[2] Beavers, R. and Hampson, R. (2000) The Beavers Systems Model of Family Functioning. The Association for Family Therapy, No. 22, 128-143.

[3] Miller, I.W., Ryan, C.E., et al. (2000) The McMaster Approach to Families: Theory, Assessment, Treatment and Research. Journal of Family Therapy, 22, 168-189. http://dx.doi.org/10.1111/1467-6427.00145

[4] Skinner, H. and Steinhauer, P. (2000) Family Assessment Measure and Process Model of Family Functioning. Journal of Family Therapy, 22, 190-210. http://dx.doi.org/10.1111/1467-6427.00146

[5] Epstein, N.B., et al. (1983) The McMaster Family Assessment Device. Journal of Marital and Family Therapy, 9, 171180. http://dx.doi.org/10.1111/j.1752-0606.1983.tb01497.x

[6] Chou, T.D., Huang, C.H., Lin, H.W., et al. (1991) A Study On the Application of Family APGAR. Chinese Journal of Family Medicine, 2, 73-79.

[7] McFarlane, A.H., Bellissimo, A., et al. (1995) Family Structure, Family Functioning and Adolescent Well-Being: The Transcendent Influence of Parental Style. Journal of Child and Psychology and Psychiatry, 36, 847-864. http://dx.doi.org/10.1111/j.1469-7610.1995.tb01333.x

[8] Andrew, L.S. (1976) Crisis Theory and Family Growth. The Family Coordinator, 7, 291-295. 